\title{
Could this be another story of montage?
}

\author{
Sung Uk Choi \\ Department of Anesthesiology and Pain Medicine, Korea University College of Medicine, Seoul, Korea
}

In October 1996, the bispectral index monitor (BIS) was introduced to anesthesiologists as a reliable brain function monitor that allowed anesthetic titration over the complete range of cortical activity [1]. In January 2004, the BIS received Food and Drug Administration (FDA) approval for reducing the incidence of intraoperative awareness during general anesthesia [2].

The BIS algorithm has undergone a number of revisions since 1992 (v1.0). The latest commercially available BIS software (v 4.1 in the unilateral BIS and 1.4 in the bilateral BIS, Covidien, Dublin, Ireland) uses a four-lead (or six-lead) sensor placed on the forehead, and this version shows improved reliability in the sedative range with enhanced artifact recognition/rejection.

In brief, the standard BIS uses a bifrontal montage to evaluate the anesthetic depth on the basis of electroencephalography (EEG). However, this sensor placement is not possible in neurosurgical or plastic procedures that require a frontal approach because the standard application of the BIS sensor would lie in the operative field or interfere with the procedures.

Several alternative BIS montages have been studied previously. One study that compared the fronto-central (Fp1 and Fp2 to $\mathrm{CZ}$ ) and bifrontal (FPz-At) montages of the BIS found that the trend among the fronto-central placement positions was useful in monitoring the depth of anesthesia; however, the BIS values showed less utility when making comparisons between patients or as a single value [3]. Unfortunately, such frontocentral placement of the BIS sensors would interfere with the operative field in neurosurgical procedures. In addition, a study using an occipital montage specifically for neurosurgical procedures found poor agreement between the frontal and occipital values [4]. Previous versions of the BIS software, however, showed strong correlation between the different placements
$[5,6]$. A recent study comparing the standard BIS montage with an alternate BIS montage across the nasal bridge demonstrated appropriate agreement between the two montage positions [7]. However, a drawback to a nasal montage would be the requirement for increased eye protection and vigilance because of the close proximity to the inferior aspect of the eye.

The mandibular position represents an alternative sensor placement in cases in which the bifrontal setup is not possible [8]. Lee et al. demonstrated that the mandibular position could represent an alternative position during the anesthesia maintenance period; however, overall BIS values did not show agreement between the standard position and the mandibular position. In addition to the limitations mentioned by the authors, other important limitations include the fact that the mandibular area was not part of the EEG-generating components of the montage but was, rather, higher electromyographic area. All EEG-based monitors currently available, including the BIS for anesthetic depth, had time delays in reacting to anesthesia level changes. The time delay between the mandibular montage and the standard bifrontal montage would be longer than usual. Therefore, the alternative mandibular montage should be used cautiously in neurosurgical cases, in conjunction with additional, standard clinical tools for assessing anesthetic depth.

Although the utility and efficacy of the BIS has been debated [9-14], the BIS is an option for avoiding excessively light or deep anesthesia in patients at high risk for adverse outcomes from general anesthesia, and is especially useful for total intravenous anesthesia.

The limitations identified in studies evaluating alternative BIS sensor positions provide useful information for developing future algorithms or sensors for improved BIS reliability.

Corresponding author: Sung Uk Choi, M.D., Ph.D., Department of Anesthesiology and Pain Medicine, Korea University College of Medicine, 73, Inchon-ro, Seongbuk-gu, Seoul 136-705, Korea. Tel: 82-2-920-5771, Fax: 82-2-928-2275, E-mail: drchois@korea.ac.kr

(c) This is an open-access article distributed under the terms of the Creative Commons Attribution Non-Commercial License (http:// creativecommons.org/licenses/by-nc/3.0/), which permits unrestricted non-commercial use, distribution, and reproduction in any medium, provided the original work is properly cited. 


\section{References}

1. Sigl JC, Chamoun NG. An introduction to bispectral analysis for the electroencephalogram. J Clin Monit 1994; 10: $392-404$.

2. Johansen JW. Update on bispectral index monitoring. Best Pract Res Clin Anaesthesiol 2006; 20: 81-99.

3. Hall JD, Lockwood GG. Bispectral index: comparison of two montages. Br J Anaesth 1998; 80: 342-4.

4. Dahaba AA, Xue JX, Zhao GG, Liu QH, Xu GX, Bornemann H, et al. BIS-vista occipital montage in patients undergoing neurosurgical procedures during propofol-remifentanil anesthesia. Anesthesiology 2010; 112: 645-51.

5. Glass PS, Bloom M, Kearse L, Rosow C, Sebel P, Manberg P. Bispectral analysis measures sedation and memory effects of propofol, midazolam, isoflurane, and alfentanil in healthy volunteers. Anesthesiology 1997; 86: 836-47.

6. Shiraishi T, Uchino H, Sagara T, Ishii N. A comparison of frontal and occipital bispectral index values obtained during neurosurgical procedures. Anesth Analg 2004; 98: 1773-5.

7. Nelson P, Nelson JA, Chen AJ, Kofke WA. An alternative position for the BIS-Vista montage in frontal approach neurosurgical cases. J Neurosurg Anesthesiol 2013; 25: 135-42.

8. Lee SY, Kim YS, Lim BG, Kim H, Kong MH, Lee IO. Comparison of bispectral index scores from the standard frontal sensor position with those from an alternative mandibular position. Korean J Anesthesiol 2014; 66: 267-73.

9. Punjasawadwong Y, Boonjeungmonkol N, Phongchiewboon A. Bispectral index for improving anaesthetic delivery and postoperative recovery. Cochrane Database Syst Rev 2007: (4): CD003843.

10. Avidan MS, Jacobsohn E, Glick D, Burnside BA, Zhang L, Villafranca A, et al. Prevention of intraoperative awareness in a high-risk surgical population. N Engl J Med 2011; 365: 591-600.

11. Klopman MA, Sebel PS. Cost-effectiveness of bispectral index monitoring. Curr Opin Anaesthesiol 2011; 24: 177-81.

12. Mashour GA, Shanks A, Tremper KK, Kheterpal S, Turner CR, Ramachandran SK, et al. Prevention of intraoperative awareness with explicit recall in an unselected surgical population: a randomized comparative effectiveness trial. Anesthesiology 2012; 117: 717-25.

13. Villafranca A, Thomson IA, Grocott HP, Avidan MS, Kahn S, Jacobsohn E. The impact of bispectral index versus end-tidal anesthetic concentration-guided anesthesia on time to tracheal extubation in fast-track cardiac surgery. Anesth Analg 2013; 116: 541-8.

14. Zand F, Hadavi SM, Chohedri A, Sabetian P. Survey on the adequacy of depth of anaesthesia with bispectral index and isolated forearm technique in elective Caesarean section under general anaesthesia with sevoflurane. Br J Anaesth 2014 [Epub ahead of print]. 